PAPER

\section{Three-dimensional background field gravity: a Hamilton--Jacobi analysis}

To cite this article: N T Maia et al 2015 Class. Quantum Grav. 32185013

View the article online for updates and enhancements.

\section{Related content}

- Hamilton--Jacobi formalism for linearized
$\frac{\text { gravity }}{\text { M C Bertin, B M Pimentel, C E Valcárcel et }}$
al.
- Hamiltonian analysis of Plebanski theory
E Buffenoir, M Henneaux, K Noui et al.
- Constraint structure of O(3) nonlinear
$\frac{\text { sigma model revisited }}{\text { Soon-Tae Hong, Yong-Wan Kim, Young- }}$
Jai Park et al.

\section{Recent citations}

- $\frac{\text { Hamilton-Jacobi analysis of the four- }}{\text { dimensional BF model with cosmological }}$

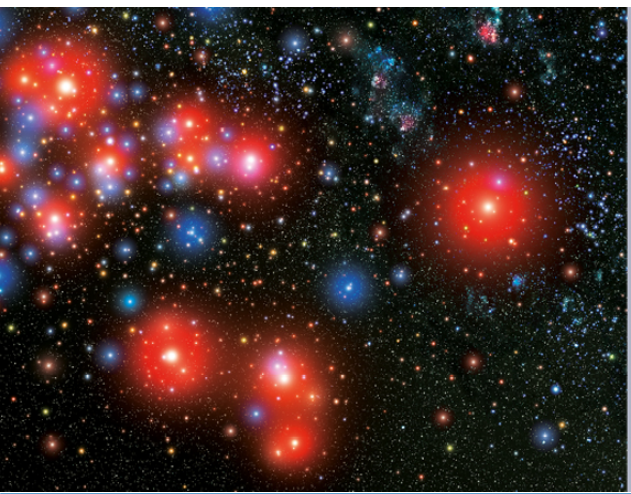

A.A

Part of your publishing universe and your first choice for astronomy, astrophysics, solar physics and planetary science ebooks. iopscience.org/books/aas 


\title{
Three-dimensional background field gravity: a Hamilton-Jacobi analysis
}

\author{
N T Maia ${ }^{1}$, B M Pimentel ${ }^{1}$ and C E Valcárcel ${ }^{2,3}$ \\ ${ }^{1}$ Instituto de Física Teórica, UNESP—São Paulo State University, PO Box 70532-2, \\ 01156-970, São Paulo, SP, Brazil \\ ${ }^{2}$ Centro de Matemática, Computação e Cognição, Universidade Federal do ABC, \\ 09210-170 Santo André, SP, Brazil
}

E-mail: nmaia@ift.unesp.br, pimentel@ift.unesp.br and valcarcel.flores@gmail.com

Received 18 April 2015

Accepted for publication 3 August 2015

Published 2 September 2015

\begin{abstract}
We analyse the constraint structure of the background field model for threedimensional gravity including a cosmological term via the Hamilton-Jacobi formalism. We find the complete set of involutive Hamiltonians that assures the integrability of the system and calculate the characteristic equations of the system. We established the equivalence between these equations and the field equations and also obtain the generators of canonical and gauge transformations.
\end{abstract}

Keywords: constrained systems, Hamilton-Jacobi formalism, background field

\section{Introduction}

Topological quantum field theories were introduced by Witten [1] in the late 80s and until now they have found a wide range of applications in physics. One characteristic of these theories is that their correlation functions do not depend on the spacetime metric. According to Birmingham [2] the topological quantum field theories can be divided into two groups: the Witten (or cohomological) type and the Schwarz type. The Chern-Simons gauge theory is a Schwarz type topological quantum field theory defined in odd dimensions which is used, for example, in addition to three-dimensional kinetic actions to build the so-called topologically massive theories [3].

The background field (BF) model is another Schwarz type topological quantum field theory and had been widely used due to its relation with gravity. For example, it has been shown that the two-dimensional BF model can be equivalent to the two-dimensional Jackiw-

3 Author to whom any correspondence should be addressed. 
Teitelboim gravity [4] for a given gauge group [5]. The three-dimensional BF model is equivalent to the first order formulation of pure general relativity under the Lorentz gauge group $S O(2,1)$ [6] and the four-dimensional gravity is equivalent to the Plebanski action [7] which consists of a BF action plus a Lagrangian multiplier. An extensive review between these equivalences can be found in [8].

The BF lower dimensional models of gravity are good laboratories for the study of spin foam quantization [9] and loop quantum gravity. In both schemes of quantization, the simplectic structure of the BF model is of utmost importance. In order to identify the correct phase space, the Dirac canonical analysis [10] is one of the most used tools. This analysis has been done in two- [11] and three- [12] dimensional BF models of gravity. Nonetheless, there are other schemes of constraint analysis, such as the Faddeev-Jackiw [13] formalism and the Hamilton-Jacobi formalism.

A first attempt to use the Hamilton-Jacobi formalism as an approach to constrained systems was given by Dominici et al [14]. Here we will deal with the approach developed by Güler [15] as an extension to Carathéodory's equivalent Lagrangians method for the calculus of variations [16]. The conditions for stationary action are reduced to a set of HamiltonJacobi partial differential equations, also called Hamiltonians, that must obey the Frobenius integrability condition. In [17] has been shown that in order to satisfy the integrability condition the non-involutive Hamiltonians must be eliminated, and this way they redefine the dynamic of the system by building a generalised bracket. Therefore, we end up with a set of complete involutive Hamiltonians, which plays the role of generators of the canonical transformations [18]. The Hamilton-Jacobi formalism has been generalised to higher order Lagrangians and Berezin systems, among others [19], as well as applied to different kind of physical systems, more recently to topologically massive theories [20] and gravity models [21], including the two-dimensional BF gravity [22]. In this article we will apply the Hamilton-Jacobi formalism to the three-dimensional BF model for gravity.

In the following section we will show the Hamilton-Jacobi formalism (for a more detailed explanation see $[17,18])$. In section 3 the three-dimensional BF gravity will be presented. In section 4 we will perform its Hamilton-Jacobi constraint analysis and build the generalised brackets. In section 5 we will compute the characteristic equations and analyse the dynamical evolution along the independent parameters of the theory. From this analysis we obtain the equivalence between the Lagrangian equations of motion and the temporal evolution of the CE. From the evolution along the parameters related to the involutive Hamiltonians, we obtain the generators of canonical and gauge transformations. In section 6 we will discuss the results.

\section{The Hamilton-Jacobi formalism}

Let us consider a physical system with a Lagrangian function $L=L\left(x^{i}, \dot{x}^{i}, t\right)$, where the Latin indices $i, j$ go from 1 to $n$, with $n$ being the dimension of the configuration space. This Lagrangian is called singular or constrained if it does not satisfy the Hessian condition, which states that the matrix elements $W_{i j}=\frac{\partial^{2} L}{\partial \dot{x}^{i} \partial \dot{x}^{j}}$ have a determinant equal to zero. Whenever the Hessian condition (det $W_{i j} \neq 0$ ) is not satisfied, it is implied that some of the conjugated momenta $p_{i}=\frac{\partial L}{\partial \dot{x}^{i}}$ are not invertible on the velocities. By considering $k$ non-invertible momenta and $m=n-k$ invertible momenta, we have 


$$
p_{z}-\frac{\partial L}{\partial \dot{x}^{z}}=0
$$

where $z=1, \ldots, k$. Defining $H_{z} \equiv-\frac{\partial L}{\partial \dot{x}^{z}}$, the above equation is rewritten as

$$
H_{z}^{\prime} \equiv p_{z}+H_{z}=0 \text {. }
$$

We call the constraints represented in this way Hamiltonians. Defining $p_{0} \equiv \frac{\partial S}{\partial t}$, the Hamilton-Jacobi equation is the Hamiltonian

$$
H_{0}^{\prime} \equiv p_{0}+H_{0}=0 \text {. }
$$

The canonical Hamiltonian function $H_{0}=p_{a} \dot{x}^{a}+p_{z} \dot{x}^{z}-L$, with $a=1, \ldots, m$, does not depend on the non-invertible velocities $\dot{x}^{z}$ if the constraints are carried out. Putting together (2) and (3), we form the initial set of Hamilton-Jacobi partial differential equations:

$$
H_{\alpha}^{\prime} \equiv p_{\alpha}+H_{\alpha}=0,
$$

where $\alpha=0,1, \ldots, k$. Through Cauchy's method [16], the characteristic equations related to the first order equations system (4) are given by

$$
\mathrm{d} x^{a}=\frac{\partial H_{\alpha}^{\prime}}{\partial p_{a}} \mathrm{~d} t^{\alpha}, \quad \mathrm{d} p_{a}=-\frac{\partial H_{\alpha}^{\prime}}{\partial x^{a}} \mathrm{~d} t^{\alpha}, \quad \mathrm{d} S=\left(p_{a} \mathrm{~d} x^{a}-H_{\alpha} \mathrm{d} t^{\alpha}\right) .
$$

From these differential equations, the Poisson brackets defined on the extended phase space $\left(x^{a}, t^{\alpha}, p_{a}, p_{\alpha}\right)$ can be used to express in a concise form the evolution of any function $f=f\left(x^{a}, t^{\alpha}, p_{a}, p_{\alpha}\right)$ :

$$
\mathrm{d} f=\left\{f, H_{\alpha}^{\prime}\right\} \mathrm{d} t^{\alpha} .
$$

This is the fundamental differential whereby the Hamiltonians can be seen as the generators of the dynamical evolution of the phase space functions.

A geometrical interpretation can be given at this point. The solutions of the first two equations of (5) give rise to a congruence of curves on the reduced phase space $\left(x^{a}, p_{a}\right)$. The characteristic curves $x^{a}\left(t, x^{z}\right)$ describe the dynamical trajectories and depend on the $k+1$ parameters $t^{\alpha}$ which in turn must be regarded as the independent variables of the system. A complete solution of (4) is given by a family of surfaces orthogonal to the characteristic curves, and its existence is ensured by satisfying the Frobenius integrability condition [18] which is written as

$$
\left\{H_{\alpha}^{\prime}, H_{\beta}^{\prime}\right\}=C_{\alpha \beta}^{\gamma} H_{\gamma}^{\prime} .
$$

This means that the Hamiltonians must close a Lie algebra. Equivalently,

$$
\mathrm{d} H_{\alpha}^{\prime}=0 .
$$

Hamiltonians that satisfy the Frobenius integrability condition are called involutives while the non-involutives are those that do not satisfy it. We can add new constraints to the system by imposing condition (8) and then completing the set of Hamilton-Jacobi partial differential equations. However, sometimes this procedure is not sufficient to make the set of Hamilton-Jacobi partial differential equations integrable. When condition (8) is imposed, some Hamiltonians may provide relations that exhibit dependence between some parameters. These Hamiltonians can be used to construct a new algebra which we call the generalised brackets:

$$
\{A, B\}^{*}=\{A, B\}-\left\{A, H_{\bar{a}}^{\prime}\right\}\left(M_{\bar{a} \bar{b}}\right)^{-1}\left\{H_{\bar{b}}^{\prime}, B\right\} .
$$


The indices $\bar{a}$ and $\bar{b}$ are related to the non-involutive Hamiltonians whose parameters are somehow related. The matrix $M$ is built from the Poisson brackets of these Hamiltonians, i.e., its elements are $M_{\bar{a} \bar{b}}=\left\{H_{\bar{a}}^{\prime}, H_{\bar{b}^{\prime}}{ }^{\prime}\right.$. In this way, these non-involutive Hamiltonians are absorbed in the new algebra. The integrability of the remaining Hamiltonians must be analysed through the generalised bracket algebra instead of the Poisson bracket algebra. New Hamiltonians may be added to complete the Hamilton-Jacobi partial differential equations set in this process until we get as a result an integrable set of Hamilton-Jacobi partial differential equations.

Let us define the variables on the extended phase space as $z^{I}=\left(x^{a}, t^{\alpha}, p_{a}, p_{\alpha}\right)$ and define the vector field $X_{\alpha}$ with components

$$
X_{\alpha}^{I} \equiv\left\{z^{I}, H_{\alpha}^{\prime}\right\}^{*},
$$

such that any function on the extended phase space can be written as

$$
\mathrm{d} F=\left\{F, H_{\alpha}^{\prime}\right\}^{*} \mathrm{~d} t^{\alpha}=X_{\alpha}[F] \mathrm{d} t^{\alpha} .
$$

The vectors $X_{\alpha}$ are related to the dynamical evolution of the system, since the characteristic equations are included in (10). From the definition of vectors $X_{\alpha}$ and using the Jacobi Identity we obtain

$\left[X_{\alpha}, X_{\beta}\right] F=\left\{\left\{F, H_{\beta}^{\prime}\right\}^{*}, H_{\alpha}^{\prime}\right\}^{*}-\left\{\left\{F, H_{\alpha}^{\prime}\right\}^{*}, H_{\beta}^{\prime}\right\}^{*}=\left\{\left\{H_{\alpha}^{\prime}, H_{\beta}^{\prime}\right\}^{*}, F\right\}^{*}$.

Whenever the system is integrable, i.e., when (7) or (8) is valid, we can write

$$
\left[X_{\alpha}, X_{\beta}\right] F=-\left\{f_{\alpha \beta}^{\gamma} H_{\gamma}^{\prime}, F\right\}^{*}=f_{\alpha \beta}^{\gamma} X_{\gamma}[F]-\left\{f_{\alpha \beta}^{\gamma}, F\right\}^{*} H_{\gamma}^{\prime},
$$

where $f_{\alpha \beta}^{\gamma}=-C_{\alpha \beta}^{\gamma}$. If the structure constants are independent of the variables of the extended phase space the integrability condition becomes a condition over the commutator

$$
\left[X_{\alpha}, X_{\beta}\right]=f_{\alpha \beta}^{\gamma} X_{\gamma},
$$

which is, indeed, the necessary condition for $X_{\alpha}$ to be a complete basis.

In general, a transformation of a function $F$ can be written as

$$
\delta F=\delta t^{\alpha} X_{\alpha} F
$$

where $\delta t^{\alpha}=\bar{t}^{\alpha}-t^{\alpha}$ are arbitrary functions of $z^{I}$. However, note that if we choose $\delta t^{\alpha}=\mathrm{d} t^{\alpha}$, equation (14) becomes the fundamental differential. For any variable of the extended phase space $z^{I}$ we have

$$
\delta z^{I}=\bar{z}^{I}\left(\bar{t}^{\alpha}\right)-z^{I}\left(t^{\alpha}\right)=\delta t^{\alpha} X_{\alpha}\left[z^{I}\right] .
$$

Now, let us consider a transformation $g$ such that

$$
\bar{z}^{I}\left(\bar{t}^{\alpha}\right)=g z^{I}\left(t^{\alpha}\right) .
$$

In this case

$$
g=1+\delta t^{\alpha} X_{\alpha}
$$

We say that transformation $g$ carries the infinitesimal flows generated by the vectors $X_{\alpha}$. These are what we call characteristic flows. It can be shown that whenever the integrability condition is satisfied, the transformation $g$ has an inverse

$$
g^{-1}=1-\delta t^{\alpha} X_{\alpha}
$$


and also preserves the symplectic structure $\omega \equiv \mathrm{d} x^{a} \wedge \mathrm{d} p_{a}+\mathrm{d} t^{\alpha} \wedge \mathrm{d} p_{\alpha}+\mathrm{d} H_{\alpha} \wedge \mathrm{d} t^{\alpha}$

$$
g \omega g^{-1}=\omega .
$$

This shows that $g$ are canonical transformations and that the complete set of involutive Hamiltonians $H_{\alpha}^{\prime}$ are the generators of these transformations.

In order to relate the canonical transformations with the gauge ones, we need to restrict the study to fixed times $\delta t^{0}=\delta t=0$, which is the classical equivalent to a fixed point transformation in field theory. The transformation on any variable $z^{I}$ now reads

$$
\delta z^{I}=\left\{z^{I}, H_{z}^{\prime}\right\}^{*} \delta t^{z},
$$

If we can keep this transformation canonical, the integrability condition must be satisfied, that is,

$$
\left\{H_{x}^{\prime}, H_{y}^{\prime}\right\}^{*}=C_{x y}^{z} H_{z}^{\prime}
$$

Nonetheless, this condition does not guarantee the integrability on the algebra of the Hamiltonians, which is

$$
\left\{H_{x}^{\prime}, H_{y}^{\prime}\right\}^{*}=C_{x y}^{0} H_{0}^{\prime}+C_{x y}^{z} H_{z}^{\prime} .
$$

To conciliate both equations we must consider whether $C_{x y}^{0}=0$ or $H_{0}^{\prime}=0$. However, condition $C_{x y}^{0}=0$ is too strong since it implies that $\left\{H_{0}^{\prime}, H_{z}^{\prime}\right\}=0$, which is almost never satisfied. On the other hand, the condition $H_{0}^{\prime}=0$ constrains the phase space. Under this assumption, we define

$$
G^{\mathrm{can}} \equiv H_{z}^{\prime} \delta t^{z},
$$

which is the generator of the canonical transformations, once

$$
\delta z^{I}=\left\{z^{I}, G^{\mathrm{can}}\right\}^{*}
$$

\section{Three-dimensional BF model}

Let us consider a $d$-dimensional manifold $\mathcal{M}$, a Lie group $G$, a connection $A$ and a $(d-2)$ form $B$ called the background field. With those elements let us build the following action

$$
W_{B F}=\int_{\mathcal{M}} \operatorname{tr}[B \wedge F]
$$

where $F$ is the curvature of the connection $A$, i.e., $F=D A$. Due to the properties of the trace and the exterior product $\wedge$ it is straightforward to see that this action is gauge invariant.

In three dimensions we can add another invariant, $\operatorname{tr}[B \wedge B \wedge B]$. Therefore, the threedimensional BF action can be written as

$$
W_{B F}=\int_{\mathcal{M}} \operatorname{tr}(B \wedge F(A)+\kappa B \wedge B \wedge B),
$$

where $\kappa$ is a constant. Due to its construction, (26) is invariant under gauge transformation:

$$
\delta A=D \chi, \quad \delta B=[B, \chi],
$$

but also quasi-invariant under shift transformation:

$$
\delta B=D \eta, \quad \delta A=3 \kappa[B, \eta],
$$

with $\xi$ and $\eta$ being arbitrary functions. 
It has been shown that in three dimensions and considering $G$ as the Lorentz group $S O(1,2)$, the BF action (26) is equivalent to Einstein-Hilbert-Palatini gravity in terms of vielbeins. Therefore, considering $G$ as the Lorentz group and $\kappa=-\Lambda / 3$, the action (26) represents Riemann gravity plus the cosmological constant.

Before we proceed with any kind of quantization scheme, the reduced phase space of the system must be well defined. The true degrees of freedom are determined after the analysis of the constraints of the theory.

\section{The Hamilton-Jacobi analysis of the 3D BF gravity}

The constraint analysis is not covariant. We refer to one specific time choice to build the Hamilton-Jacobi equations. It is then appropriate to leave the differential form notation and write the Lagrangian in terms of the components of the background and gauge field, i.e.,

$$
A=A_{\mu}^{a} J_{a} \mathrm{~d} x^{\mu}, B=B_{\mu}^{a} J_{a} \mathrm{~d} x^{\mu},
$$

where $J_{a}$ are generators of the $G=S O(1,2)$ group. These generators satisfy $\left[J_{a}, J_{b}\right]=f_{a b}{ }^{c} J_{c}$ and $\operatorname{tr}\left(J_{a} J_{b}\right)=\frac{1}{2} \eta_{a b}$, where $\eta_{a b}=\operatorname{diag}(+,-,-)$. Therefore

$$
\mathcal{L}=\frac{1}{2} \epsilon^{\mu \gamma \nu}\left(B_{a \mu} F_{\gamma \nu}^{a}-\frac{\Lambda}{3} f_{a b c} B_{\mu}^{a} B_{\gamma}^{b} B_{\nu}^{c}\right),
$$

where $F_{\mu \nu}^{a}=\partial_{\mu} A_{\nu}^{a}-\partial_{\nu} A_{\mu}^{a}+f_{b c}^{a} A_{\mu}^{b} A_{\nu}^{c}$. The equations of motion are

$$
\begin{aligned}
& 0=\epsilon^{\mu \gamma \nu}\left(F_{\gamma \nu}^{a}-\Lambda f_{b c}^{a} B_{\gamma}^{b} B_{\nu}^{c}\right), \\
& 0=\epsilon^{\mu \gamma \nu} D_{\gamma} B_{\nu}^{a} .
\end{aligned}
$$

Here we have made use of the definition of the covariant derivative

$$
D_{\mu} \theta_{\nu}^{a} \equiv \partial_{\mu} \theta_{\nu}^{a}+f_{b c}^{a} A_{\mu}^{b} \theta_{\nu}^{c}
$$

Furthermore, equation (31) represents the dynamical equation of three-dimensional gravity, and (32) represents the zero torsion condition.

Now, to begin with the Hamilton-Jacobi analysis of the three-dimensional BF gravity, we compute the momenta $\pi^{a}$ and $\Pi^{a}$ conjugated to $A_{\mu}^{a}$ and $B_{\mu}^{a}$, respectively,

$$
\begin{aligned}
& \pi_{a}^{\mu} \equiv \frac{\partial \mathcal{L}}{\partial \partial_{0} A_{\mu}^{a}}=\epsilon^{0 \mu \nu} B_{a \nu}, \\
& \Pi_{a}^{\mu} \equiv \frac{\partial \mathcal{L}}{\partial \partial_{0} B_{\mu}^{a}}=0 .
\end{aligned}
$$

The expressions above do not depend on any velocities $\partial_{0} A_{\mu}^{a}, \partial_{0} B_{\mu}^{a}$. Therefore they are canonical constraints of the theory. It turns out that the canonical Hamiltonian density is given by

$$
\mathcal{H}_{0}=-\epsilon^{0 \gamma \nu}\left[A_{a 0} D_{\gamma} B_{\nu}^{a}+B_{a 0}\left(F_{\gamma \nu}^{a}-\Lambda f_{b c}^{a} B_{\gamma}^{b} B_{\nu}^{c}\right)\right] .
$$

Let us define $\pi \equiv \partial_{0} S$. Then, the initial set of Hamilton-Jacobi partial differential equations is

$$
\mathcal{H}^{\prime} \equiv \pi+\mathcal{H}_{0}=0,
$$




$$
\begin{aligned}
& \mathcal{A}_{a}^{\prime 0} \equiv \pi_{a}^{0}=0, \\
& \mathcal{A}_{a}^{\prime 1} \equiv \pi_{a}^{1}-B_{a 2}=0, \\
& \mathcal{A}_{a}^{\prime 2} \equiv \pi_{a}^{2}+B_{a 1}=0, \\
& \mathcal{B}_{a}^{\prime \mu} \equiv \Pi_{a}^{\mu}=0 .
\end{aligned}
$$

The first Hamiltonian $\mathcal{H}^{\prime}$ is associated with the time parameter $t \equiv x_{0}$. The Hamiltonians $\mathcal{A}_{a}^{\prime \mu}$ arose from the non-invertible momenta $\pi_{a}^{\mu}$ and are related to the parameters $\lambda_{\mu}^{a} \equiv A_{\mu}^{a}$. Analogously, the Hamiltonians $\mathcal{B}_{a}^{\prime \mu}$ are referred to the parameters $\epsilon_{\mu}^{a} \equiv B_{\mu}^{a}$.

The fundamental Poisson brackets of the model are

$$
\begin{aligned}
& \left\{A_{\mu}^{a}(x), \pi_{b}^{\nu}\left(x^{\prime}\right)\right\}=\delta_{b}^{a} \delta_{\mu}^{\nu} \delta^{2}\left(\mathbf{x}-\mathbf{x}^{\prime}\right), \\
& \left\{B_{\mu}^{a}(x), \Pi_{b}^{\nu}\left(x^{\prime}\right)\right\}=\delta_{b}^{a} \delta_{\mu}^{\nu} \delta^{2}\left(\mathbf{x}-\mathbf{x}^{\prime}\right) .
\end{aligned}
$$

The fundamental differential characterizes the evolution of any function of the phase space. It is expressed as

$$
\begin{aligned}
\mathrm{d} f(x)= & \int\left(\left\{f(x), \mathcal{H}^{\prime}\left(x^{\prime}\right)\right\} \mathrm{d} t+\left\{f(x), \mathcal{A}_{a}^{\prime \mu}\left(x^{\prime}\right)\right\} \mathrm{d} \lambda_{\mu}^{a}\right. \\
& \left.+\left\{f(x), \mathcal{B}_{a}^{\prime \mu}\left(x^{\prime}\right)\right\} \mathrm{d} \epsilon_{\mu}^{a}\right) \mathrm{d}^{2} x^{\prime} .
\end{aligned}
$$

Now we check the integrability of the Hamilton-Jacobi partial differential equations. When the IC is applied to the Hamiltonians $\mathcal{A}_{a}^{\prime 1}, \mathcal{A}_{a}^{\prime 2}, \mathcal{B}_{a}^{\prime 1}$ and $\mathcal{B}_{a}^{\prime 2}$ we get relations of dependence between the parameters related to them. This information tells us that these Hamiltonians are non-involutive and can be used to construct the generalised brackets.

Let us rename $h_{a}^{0} \equiv \mathcal{A}_{a}^{\prime 1}, \quad h_{a}^{1} \equiv \mathcal{A}_{a}^{\prime 2}, \quad h_{a}^{2} \equiv \mathcal{B}_{a}^{\prime 1}$ and $h_{a}^{3} \equiv \mathcal{B}_{a}^{\prime 2}$. Let us denote $I, J=0,1,2,3$ as the indices of the elements of the matrix $M_{a b}^{I J}(x, y) \equiv\left\{h_{a}^{I}(x), h_{b}^{J}(y)\right\}$. We have

$$
M(x, y)=\left(\begin{array}{cccc}
0 & 0 & 0 & -1 \\
0 & 0 & 1 & 0 \\
0 & -1 & 0 & 0 \\
1 & 0 & 0 & 0
\end{array}\right) \delta^{a b} \delta^{2}\left(\mathbf{x}-\mathbf{x}^{\prime}\right) .
$$

This matrix has an inverse

$$
M^{-1}(x, y)=\left(\begin{array}{cccc}
0 & 0 & 0 & 1 \\
0 & 0 & -1 & 0 \\
0 & 1 & 0 & 0 \\
-1 & 0 & 0 & 0
\end{array}\right) \delta^{a b} \delta^{2}\left(\mathbf{x}-\mathbf{x}^{\prime}\right)
$$

and with this inverse we define the generalised brackets as

$\left\{f(x), g\left(x^{\prime}\right)\right\}^{*}=\left\{f(x), g\left(x^{\prime}\right)\right\}-\int\left\{f(x), h_{c}^{I}(y)\right\}\left[M^{-1}\left(y, y^{\prime}\right)\right]_{I J}^{c d}\left\{h_{d}^{J}\left(y^{\prime}\right), g\left(x^{\prime}\right)\right\} \mathrm{d} y \mathrm{~d} y^{\prime}$.

We can use this expression to find the fundamental generalised brackets of the theory. The non-vanishing results are given below:

$$
\left\{A_{\mu}^{a}(x), \pi_{b}^{\nu}\left(x^{\prime}\right)\right\}^{*}=\delta_{b}^{a} \delta_{\mu}^{\nu} \delta^{2}\left(\mathbf{x}-\mathbf{x}^{\prime}\right),
$$




$$
\begin{aligned}
& \left\{B_{0}^{a}(x), \Pi_{b}^{0}\left(x^{\prime}\right)\right\}^{*}=\delta_{b}^{a} \delta^{2}\left(\mathbf{x}-\mathbf{x}^{\prime}\right), \\
& \left\{A_{\mu}^{a}(x), B_{\nu}^{b}\left(x^{\prime}\right)\right\}^{*}=\delta^{a b} \epsilon_{0 \mu \nu} \delta^{2}\left(\mathbf{x}-\mathbf{x}^{\prime}\right) .
\end{aligned}
$$

Comparing with the original Poisson brackets (42), (43), we note that $B_{a}^{i}, \Pi_{i}^{a}$ are no longer conjugated variables. In fact, $B_{a}^{1}$ now plays the role of $-\pi_{a}^{2}$ and $B_{a}^{2}$ the role of $\pi_{a}^{1}$. Only $B_{0}^{a}(x), \Pi_{a}^{0}$ and $A_{\mu}^{a}, \pi_{b}^{\nu}$ remain as conjugated variables.

After building the generalised brackets, the fundamental differential (44) now takes the form

$$
\begin{aligned}
\mathrm{d} f(x)= & \int\left(\left\{f(x), \mathcal{H}^{\prime}\left(x^{\prime}\right)\right\}^{*} \mathrm{~d} t+\left\{f(x), \mathcal{A}_{a}^{\prime 0}\left(x^{\prime}\right)\right\}^{*} \mathrm{~d} \lambda_{0}^{a}\right. \\
& \left.+\left\{f(x), \mathcal{B}_{a}^{\prime 0}\left(x^{\prime}\right)\right\}^{*} \mathrm{~d} \epsilon_{0}^{a}\right) \mathrm{d}^{2} x^{\prime} .
\end{aligned}
$$

We still need to analyse the integrability condition of the Hamiltonians $\mathcal{A}_{a}^{\prime 0}$ and $\mathcal{B}_{a}^{\prime 0}$. By imposing $d \mathcal{A}_{a}^{\prime 0}=0$ and $d \mathcal{B}_{a}^{\prime 0}=0$ we note that we need to introduce two new Hamiltonians:

$$
\begin{aligned}
\mathcal{C}^{a} a & \equiv \epsilon^{0 \gamma \nu} D_{\gamma} B_{\nu}^{a}=0, \\
\mathcal{D}^{\prime a} & \equiv \frac{1}{2} \epsilon^{0 \gamma \nu}\left[F_{\gamma \nu}^{a}-\Lambda f^{a}{ }_{b c} B_{\gamma}^{b} B_{\nu}^{c}\right]=0 .
\end{aligned}
$$

Note that the canonical Hamiltonian (36) now can be written as $\mathcal{H}_{0}=-A_{a 0} \mathcal{C}^{\prime a}-B_{a 0} \mathcal{D}^{\prime a}$. The fields $\mathcal{A}_{a}^{\prime 0}$ and $\mathcal{B}_{a}^{\prime 0}$ have the role of Lagrange multipliers since they are coefficients of the constraints in the canonical Hamiltonian. The new constraints also satisfy the integrability condition and there is no need to introduce new constrains or redefine the algebra. The integrability programme is then achieved and the complete set of involutive Hamiltonians is $\mathcal{A}_{a}^{\prime 0}, \mathcal{B}_{a}^{\prime 0}, \mathcal{C}^{\prime a}, \mathcal{D}^{\prime a}$.

Let us define

$$
\begin{aligned}
& C^{\prime a}(\alpha) \equiv \int \alpha(y) \mathcal{C}^{\prime a}(y) \mathrm{d}^{2} y, \\
& D^{\prime a}(\beta) \equiv \int \beta(y) \mathcal{D}^{\prime a}(y) \mathrm{d}^{2} y,
\end{aligned}
$$

where $\alpha$ and $\beta$ are weight functions. The below relations follow:

$$
\begin{aligned}
& \left\{C^{\prime a}\left(\alpha_{1}\right), C^{\prime b}\left(\alpha_{2}\right)\right\}^{*}=f^{a b}{ }_{c} C^{\prime c}\left(\alpha_{1}, \alpha_{2}\right), \\
& \left\{C^{\prime a}\left(\alpha_{1}\right), D^{\prime b}\left(\beta_{1}\right)\right\}^{*}=f^{a b}{ }_{c} D^{\prime c}\left(\alpha_{1}, \beta_{1}\right), \\
& \left\{D^{\prime a}\left(\beta_{1}\right), D^{\prime b}\left(\beta_{2}\right)\right\}^{*}=-\Lambda f^{a b}{ }_{c} C^{\prime c}\left(\beta_{1}, \beta_{2}\right) .
\end{aligned}
$$

Note that for $\Lambda=0$, i.e., the pure three-dimensional gravity, the Hamiltonians satisfy the Poincarè algebra $I S O(2,1)$, and we also identify $D^{\prime a}$, which now commutes with all the other Hamiltonians as the generator of translations. For $\Lambda \neq 0$, the Hamiltonians close the AdS or dS algebra.

\section{Characteristic equations of the 3D BF gravity}

The integrability condition allows us to find the complete set of involutive Hamiltonians: $\mathcal{A}_{a}^{\prime 0}, \mathcal{B}_{a}^{\prime 0}, \mathcal{C}^{\prime a}, \mathcal{D}^{\prime a}$, all of which play a role in the evolution of the systems and must be added 
in the fundamental differential. Let us rename

$$
\begin{aligned}
& \mathcal{H}_{a}^{\prime 0} \equiv \mathcal{A}_{a}^{\prime 0} \quad \longrightarrow \quad \omega_{a}^{0}, \\
& \mathcal{H}_{a}^{\prime 1} \equiv \mathcal{B}_{a}^{\prime 0} \quad \longrightarrow \quad \omega_{a}^{1}, \\
& \mathcal{H}_{a}^{\prime 2} \equiv \mathcal{C}_{a}^{\prime} \quad \longrightarrow \quad \omega_{a}^{2}, \\
& \mathcal{H}_{a}^{\prime 3} \equiv \mathcal{D}_{a}^{\prime} \quad \longrightarrow \quad \omega_{a}^{3},
\end{aligned}
$$

where the $\omega_{a}$ are the respective parameters. The final form of the fundamental differential is

$$
\mathrm{d} f(x)=\int \mathrm{d} x^{\prime}\left(\left\{f(x), \mathcal{H}^{\prime}\left(x^{\prime}\right)\right\}^{*} \mathrm{~d} t+\sum_{\kappa=0}^{3}\left\{f(x), \mathcal{H}_{a}^{\prime \kappa}\left(x^{\prime}\right)\right\}^{*} \mathrm{~d} \omega_{a}^{\kappa}\right) .
$$

The characteristic equations are obtained from (56) by evaluating $f$ for the fields $\left(A_{\mu}^{a}, B_{\mu}^{a}\right)$ and the momenta $\left(\pi_{a}^{\mu}, \Pi_{a}^{\mu}\right)$. For the first set we have

$\mathrm{d} A_{\mu}^{a}=\delta_{\mu}^{0} \delta^{a b} \mathrm{~d} \omega_{b}^{0}+\delta_{\mu}^{i}\left[\left(D_{i} A_{0}^{a}-\Lambda f_{b c}^{a} B_{i}^{b} B_{0}^{c}\right) \mathrm{d} t-\delta^{a b} D_{i} \mathrm{~d} \omega_{b}^{2}+\Lambda f^{a b}{ }_{c} B_{i}^{c} \mathrm{~d} \omega_{b}^{3}\right]$,

$\mathrm{d} B_{\mu}^{a}=\delta_{\mu}^{0} \mathrm{~d} \omega^{a 1}+\delta_{\mu}^{i}\left[\left(D_{i} B_{0}^{a}-f^{a}{ }_{b c} A_{0}^{b} B_{i}^{c}\right) \mathrm{d} t-f^{a b}{ }_{c} B_{i}^{b} \mathrm{~d} \omega_{c}^{2}-\delta^{a b} D_{i} \mathrm{~d} \omega_{b}^{3}\right]$,

and

$$
\begin{aligned}
\mathrm{d} \pi_{a}^{\mu}= & \epsilon^{0 \gamma \rho}\left[\delta_{0}^{\mu} D_{\gamma} B_{a \rho}-\delta_{\rho}^{\mu}\left(D_{\gamma} B_{a 0}-f_{a}^{b c} A_{b 0} B_{c \gamma}\right)\right] \mathrm{d} t \\
& +\epsilon^{0 \gamma \rho}\left\{\delta_{\gamma}^{\mu} f^{b c}{ }_{a} B_{c \rho} \mathrm{d} \omega_{b}^{2}+\delta_{\gamma}^{\mu} \delta_{a}^{b} D_{\gamma} \mathrm{d} \omega_{b}^{3}\right\}, \\
\mathrm{d} \Pi_{a}^{\mu}= & \delta_{0}^{\mu} \mathcal{H}_{a}^{\prime 3} \mathrm{~d} t .
\end{aligned}
$$

The integrability condition ensures independence between the parameters related to the involutive set of Hamilton-Jacobi partial differential equations. Therefore, since $t=x^{0}$ is one of these parameters, we can analyse the temporal evolution of the fields independently. We have

$$
\begin{aligned}
& \partial_{0} A_{\mu}^{a}=\delta_{\mu}^{i}\left(D_{i} A_{0}^{a}-\Lambda f_{b c}^{a} B_{i}^{b} B_{0}^{c}\right), \\
& \partial_{0} B_{\mu}^{a}=\delta_{\mu}^{i}\left(D_{i} B_{0}^{a}-f_{b c}^{a} A_{0}^{b} B_{i}^{c}\right) .
\end{aligned}
$$

Note that the component $\mu=0$ of these equations states that $A_{0}^{a}, B_{0}^{a}$ are time independent parameters. This reinforces the character of the Lagrange multipliers of these variables in the canonical Hamiltonian. On the other hand, the spatial components of (61) are equivalent to equation (31). Analogously, the spatial components of (62) resemble equations (32).

For the second set of characteristic equations, we have

$$
\begin{aligned}
& \partial_{0} \pi_{a}^{\mu}=\epsilon^{0 \gamma \rho}\left[\delta_{0}^{\mu} D_{\gamma} B_{a \rho}-\delta_{\rho}^{\mu}\left(D_{\gamma} B_{a 0}-f_{a}^{b c} A_{b 0} B_{c \gamma}\right)\right], \\
& \partial_{0} \Pi_{a}^{\mu}=\delta_{0}^{\mu} \mathcal{H}_{a}^{\prime 3} .
\end{aligned}
$$

Note that the temporal evolution of the component $\pi_{a}^{\mu}$ is equal to the Hamiltonian $\mathcal{H}_{2}^{\prime a}=0$, leaving $\pi_{a}^{0}$ undetermined, just as its correspondent conjugated variable $A_{0}^{a}$. For the component $\pi_{a}^{i}$, we find that its temporal evolution equation is in agreement with the definition of canonical momenta. For $\Pi_{a}^{\mu}$, we find that its temporal evolution is equal to zero. This result is in agreement with the fact that $\Pi_{a}^{0}$ is conjugated to a Lagrange multiplier and $\Pi_{a}^{i}$ is no longer a canonical variable. 


\subsection{Generators of canonical and gauge transformations}

As it was shown in section 2, the characteristic equations also give us the generator of the canonical transformations. In our case, we need to consider the variations along the independent parameters $\omega_{a}$.

$$
\begin{aligned}
\mathrm{d} A_{\mu}^{a} & =\delta_{\mu}^{0} \delta^{a b} \mathrm{~d} \omega_{b}^{0}-\delta_{\mu}^{i} \delta^{a b} D_{i} \mathrm{~d} \omega_{b}^{2}-\delta_{\mu}^{i} \Lambda f^{a b}{ }_{c} B_{i}^{c} \mathrm{~d} \omega_{b}^{3}, \\
\mathrm{~d} B_{\mu}^{a} & =\delta_{\mu}^{0} \mathrm{~d} \omega^{a 1}-\delta_{\mu}^{i} f^{a b}{ }_{c} B_{i}^{b} \mathrm{~d} \omega_{c}^{2}-\delta_{\mu}^{i} \delta^{a b} D_{i} \mathrm{~d} \omega_{b}^{3} .
\end{aligned}
$$

These expressions can be rewritten in a much simple form if we define the function

$$
G^{\text {can }} \equiv \int\left[\mathcal{H}_{0}^{\prime a} \mathrm{~d} \omega_{a}^{0}+\mathcal{H}_{1}^{\prime a} \mathrm{~d} \omega_{a}^{1}+\mathcal{H}_{2}^{\prime a} \mathrm{~d} \omega_{a}^{2}+\mathcal{H}_{3}^{\prime a} \mathrm{~d} \omega_{a}^{3}\right] \mathrm{d}^{2} x
$$

It enables us to write

$$
\begin{aligned}
& \mathrm{d} A_{\mu}^{a}=\left\{A_{\mu}^{a}, G^{\mathrm{can}}\right\}^{*}, \\
& \mathrm{~d} B_{\mu}^{a}=\left\{B_{\mu}^{a}, G^{\mathrm{can}}\right\}^{*} .
\end{aligned}
$$

As the variations of the phase space coordinates can be expressed in this way, we call $G^{\text {can }}$ the generator of canonical transformations.

On the other hand, in order to relate generator of canonical transformations with that of symmetries, we need to go further with the integrability condition. Let us consider the set of variations (65) and (66) now rewritten as

$$
\begin{aligned}
& \delta A_{\mu}^{a}=\delta_{\mu}^{0} \delta^{a b} \delta \omega_{b}^{0}-\delta_{\mu}^{i} \delta^{a b} D_{i} \delta \omega_{b}^{2}-\delta_{\mu}^{i} \Lambda f^{a b}{ }_{c} B_{i}^{c} \delta \omega_{b}^{3}, \\
& \delta B_{\mu}^{a}=\delta_{\mu}^{0} d \omega^{a 1}-\delta_{\mu}^{i} f^{a b}{ }_{c} B_{i}^{b} \delta \omega_{c}^{2}-\delta_{\mu}^{i} \delta^{a b} D_{i} \delta \omega_{b}^{3},
\end{aligned}
$$

where the variations $\delta \omega_{a}^{\kappa}$ may depend on each other. If the variations (70), (71) are symmetries of the three-dimensional BF gravity, then they must be solutions of the fixed point variation

$$
\delta \mathcal{L}=\frac{1}{2} \epsilon^{\alpha \mu \nu}\left(F_{\mu \nu}^{a}-\Lambda f_{b c}^{a} B_{\mu}^{b} B_{\nu}^{c}\right) \delta B_{\alpha}^{a}+\epsilon^{\alpha \mu \nu} B_{\mu}^{a} D_{\nu} \delta A_{a \alpha}=0 .
$$

By replacing (70)-(72) and using the Bianchi identity it follows that

$$
\begin{aligned}
\delta \mathcal{L}= & \epsilon^{i j}\left[\frac{1}{2} F_{i j}^{a}\left(\delta \omega^{a 1}+f_{a b c} B_{0}^{b} \delta \omega_{c}^{2}\right)+B_{i}^{a} D_{j}\left(D_{0} \delta \omega_{a}^{2}+\delta \omega_{a}^{0}\right)+F_{a 0 j} D_{i} \delta \omega^{a 3}\right] \\
& +-\Lambda f_{a b c} \epsilon^{i j}\left[\frac{1}{2} B_{i}^{b} B_{j}^{c}\left(\delta \omega^{a 1}+f_{n m}^{a} B_{0}^{n} \delta \omega^{m 2}\right)-B_{0}^{a} B_{j}^{c} D_{i} \delta \omega^{b 3}\right] \\
& +-\Lambda f_{a b c} \epsilon^{i j}\left[-B_{0}^{a} D_{i}\left(B_{j}^{b} \delta \omega^{c 3}\right)+B_{i}^{a} D_{0}\left(B_{j}^{b} \delta \omega^{c 3}\right)\right] .
\end{aligned}
$$

Since this is one equation for four parameters, we expect to obtain a relation between some of the $\delta \omega_{a}^{\kappa}$. A good approach to solve $\delta \mathcal{L}=0$ is by considering special cases, such as setting some of the parameters equal to zero. However, by inspection of (73), we see that $\delta \omega^{a 0}=0$ or $\delta \omega^{a 1}=0$ are not good choices for solving the equation. On the other hand, if we consider $\delta \omega^{a 3}=0$, equation (73) becomes 


$$
\begin{aligned}
\delta \mathcal{L}= & \epsilon^{i j}\left[\frac{1}{2} F_{i j}^{a}\left(\delta \omega^{a 1}+f_{a b c} B_{0}^{b} \delta \omega_{c}^{2}\right)+B_{i}^{a} D_{j}\left(D_{0} \delta \omega_{a}^{2}+\delta \omega_{a}^{0}\right)\right] \\
& +-\Lambda f_{a b c} \epsilon^{i j}\left[\frac{1}{2} B_{i}^{b} B_{j}^{c}\left(\delta \omega^{a 1}+f_{n m}^{a} B_{0}^{n} \delta \omega^{m 2}\right)\right] .
\end{aligned}
$$

Of course, we have an invariance, $\delta \mathcal{L}=0$, when we choose $\delta \omega_{a}^{0}=-D_{0} \delta \omega_{a}^{2}$ and $\delta \omega^{a 1}=-f_{b c}^{a} B_{0}^{b} \delta \omega^{c 2}$. By replacing it in the set of Hamilton-Jacobi variations, we get

$$
\begin{aligned}
\delta B_{\mu}^{a} & =-f_{b c}^{a} B_{\mu}^{b} \delta \omega^{c 2}, \\
\delta A_{a \mu} & =-D_{\mu} \delta \omega_{a}^{2} .
\end{aligned}
$$

By setting $\omega^{a 2}=-\chi^{a}$, we obtain the gauge transformation (27). These transformations are generated by

$$
G^{\text {Gauge }} \equiv \int\left[\mathcal{H}_{0}^{\prime a} D_{0}+\mathcal{H}_{1}^{\prime b} f_{b c}^{a} B_{0}^{c}-\mathcal{H}_{2}^{\prime a}\right] \delta \chi_{a}^{2} \mathrm{~d}^{2} x
$$

Now, if we set $\delta \omega^{a 2}=0$, we obtain

$$
\begin{aligned}
\delta \mathcal{L} & =\epsilon^{i j}\left[\frac{1}{2} F_{i j}^{a} \delta \omega^{a 1}+B_{i}^{a} D_{j} \delta \omega_{a}^{0}+F_{a 0 j} D_{i} \delta \omega^{a 3}\right] \\
& +-\Lambda f_{a b c} \epsilon^{i j}\left[\frac{1}{2} B_{i}^{b} B_{j}^{c} \delta \omega^{a 1}-B_{0}^{a} D_{i}\left(B_{j}^{b} \delta \omega^{c 3}\right)+B_{i}^{a} D_{0}\left(B_{j}^{b} \delta \omega^{c 3}\right)-B_{0}^{a} B_{j}^{c} D_{i} \delta \omega^{b 3}\right],
\end{aligned}
$$

which, up to boundary terms, becomes

$$
\begin{aligned}
\delta \mathcal{L}= & \epsilon^{i j}\left[-D_{j} B_{i}^{a}\left(\delta \omega_{a}^{1}-\Lambda f_{a b c} B_{0}^{b} \delta \omega^{c 3}\right)+\frac{1}{2} F_{a i j}\left(D_{0} \delta \omega^{a 3}+\delta \omega^{a 0}\right)\right] \\
& +-\epsilon^{i j} \Lambda f_{a b c}\left[\frac{1}{2} B_{i}^{a} B_{j}^{b}\left(D_{0} \delta \omega^{c 3}+\delta \omega^{c 0}\right)\right]
\end{aligned}
$$

This variation is equal to zero if we set $\delta \omega^{a 0}=-D_{0} \delta \omega^{a 3}$ and $\delta \omega_{a}^{1}=\Lambda f_{a b c} B_{0}^{b} \delta \omega^{c 3}$. Under these conditions

$$
\begin{aligned}
\delta B_{\mu}^{a} & =-D_{\mu} \delta \omega^{a 3}, \\
\delta A_{a \mu} & =\Lambda f_{a b c} B_{\mu}^{b} \delta \omega^{c 3} .
\end{aligned}
$$

The shift transformation (28) can be obtained just by setting $\eta^{a}=-\omega^{a 3}$ in the previous relations. Its correspondent generator is given by

$$
G^{\text {shift }} \equiv \int\left[\mathcal{H}_{0}^{\prime a} D_{0}-\Lambda f_{b c}^{a} \mathcal{H}_{1}^{\prime b} B_{0}^{c}-\mathcal{H}_{3}^{\prime a}\right] \delta \eta_{a}^{3} \mathrm{~d}^{2} x
$$

Therefore, we have obtained the gauge and shift transformations as well as their respective generators with the use of the Hamilton-Jacobi formalism.

\section{Final remarks}

We have used the Hamilton-Jacobi formalism to analyse the constraint structure of the threedimensional BF gravity with a cosmological constant $\Lambda$. This procedure consisted of finding the complete set of involutive Hamiltonians that generates the dynamical evolution of the system. We achieved this using the Frobenius integrability condition over the initial set of Hamilton-Jacobi partial differential equations. We noticed that there is a subgroup of Hamiltonians $\left(\mathcal{A}_{a}^{\prime \prime}, \mathcal{A}_{a}^{\prime 2}, \mathcal{B}_{1}^{\prime a}, \mathcal{B}_{2}^{\prime a}\right)$ that does not satisfy the integrability condition and with 
them we built the generalised brackets and reduced the phase space such that the system was governed by a new symplectic structure. By satisfying the integrability condition for the rest of the Hamiltonians, we found new constraints $\left(\mathcal{C}_{a}^{\prime}, \mathcal{D}_{a}^{\prime}\right)$. In the case of a cosmological constant equal to zero, these Hamiltonians satisfy the $\operatorname{ISO}(1,2)$ algebra and the Hamiltonians $\mathcal{D}_{a}^{\prime}$ commute. When the cosmological constant is not zero, the Hamiltonians satisfy the AdS or $\mathrm{dS}$ algebra.

Then, we computed the characteristic equations, which depend on the time parameter $x^{0}$ and the parameters $\omega_{a}^{\kappa}$ related to the involutive Hamiltonians. Since all the Hamiltonians satisfy the integrability condition, their correspondent parameters are linearly independent. This means that evolution along any parameter can be considered independently. As a result, we saw that the time evolution of the characteristic equations are equivalent to the field equations of BF gravity, and the evolution along the parameters $\omega_{a}^{\kappa}$ is related to the canonical transformations. Therefore, the linear combination of the four corresponding Hamiltonians gave the generator of the canonical transformations.

It was possible to relate the generator of canonical transformations with those related to the gauge and shift transformations. To achieve this, we considered the $\omega_{a}^{\kappa}$ parameters as dependent on each other. Furthermore, if they are an invariance of the theory they must eliminate, up to the boundary term, the fixed point Lagrangian variation. This way, we needed to solve an equation for four dependent variables.

As we mentioned earlier, Dirac's formalism is one of the most powerful tools to deal with constraint analysis. However, the problem of finding the gauge structure of a particular theory as a result of its constraint analysis is still a problem being discussed. Dirac's conjecture states that all of the first-class constraints generate the symmetries. Nevertheless, it is only a conjecture and some models have been found that contradict it (see for example [23]). One of the most used algorithms to build the gauge generators of a constrained theory is the one given by Castellani [24]. As we have shown, in the Hamilton-Jacobi formalism we have obtained the generators of gauge and shift transformations without the use of Castellani's algorithm. Furthermore, we have obtained all constraints from a mathematical theorem: the Frobenius integrability condition. Also note that this condition allows us to build the generalised brackets, which, even though they have a close resemblance to Dirac's brackets, are defined with only non-involutive constraints.

There is still one element that has not been fully explored yet, which is the characteristic equation that governs the dynamic of the function $S$ in (5). The integration of this characteristic equation for regular systems leads us to the action functional in the canonical form. Therefore, for constrained systems, it will be related to an extended action functional that could provide a method for quantisation via the path integral; however, the quantisation procedure is still a work in progress.

\section{Acknowledgments}

The authors thank M C Bertin for reading the manuscript and for suggestions. The authors also thank the referees for their valuable comments. NTM was supported by CAPES. BMP was partially supported by $\mathrm{CNPq}$ and CAPES. CEV was supported by FAPESP.

\section{References}

[1] Witten E 1988 Topological quantum field theory Commun. Math. Phys. 117353 
[2] Birmingham D, Blau M, Rakowski M and Thompson G 1991 Topological field theory Phys. Rept. 209129

[3] Deser S, Jackiw R and Templeton S 1982 Three-dimensional massive gauge theories Phys. Rev. Lett. 48975

Deser S, Jackiw R and Templeton S 1982 Topologically massive gauge theories Ann. Phys. 140 372

Deser S, Jackiw R and Templeton S 1988 Topologically massive gauge theories (erratum) Ann. Phys. 185406

Deser S, Jackiw R and Templeton S 2000 Topologically massive gauge theories Annals Phys. 281409

[4] Teitelboim C 1983 Supergravity and hamiltonian structure in two spacetime dimensions Phys. Lett. B126 41

Jackiw R 1984 Quantum Theory of Gravity ed S Christensen (Bristol, UK: Hilger)

Teitelboim C 1984 Quantum Theory of Gravity ed S Christensen (Bristol, UK: Hilger)

[5] Fukuyama T and Kamimura K 1985 Gauge Theory of two-dimensional gravities Phy. Lett. B 160 259

Isler K and Trugenberger C A 1989 Gauge Theory of two-dimensional quantum gravity Phys. Rev. Lett. 63834

[6] Oda I and Yahikozawa S 1994 Effective actions of (2+1)-dimensional gravity and Bf theory Class. Quantum Grav. 112653

[7] Plebanski J F 1977 On the separation of einsteinian substructures J. Math. Phys. 182511

[8] Freidel L and Speziale S 2012 On the relations between gravity and bf theories SIGMA 8032

[9] Perez A 2013 The spin foam approach to quantum gravity Living Rev. Rel. 163

[10] Dirac P A M 1950 Generalized Hamiltonian dynamics Can. J. Math. 2129

Dirac P A M 1951 The Hamiltonian form of field dynamics Can. J. Math. 31

Dirac P A M 1964 Lectures on Quantum Mechanics (New York: Yeshiva University)

[11] Constantinidis C P, Lourenço J A, Morales I, Piguet O and Rios A 2008 Canonical analysis of the Jackiw-Teitelboim model in the temporal gauge: I. The classical theory Class. Quantum. Grav. 25125003

[12] Escalante A and Tzompantzi O R 2014 Hamiltonian dynamics and gauge symmetry for threedimensional Palatini theory with cosmological constant J. High Energy. Phys. JHEP05 (2014)073

[13] Faddeev L and Jackiw R 1988 Hamiltonian reduction of unconstrained and constrained systems Phys. Rev. Lett. 601692

[14] Dominici D, Gomis J, Longhi G and Pons J M 1984 Hamilton-Jacobi theory for constrained systems J. Math. Phys. 252439

[15] Güler Y 1987 il Nuovo Cimento B 100251

Güler Y 1992 J. Math. Phys. 30785

Güler Y 1992 il Nuovo Cimento B 1071398

[16] Carathéodory C 1999 Calculus of Variations and Partial Differential equations of the First Order 3rd edn (American Mathematical Society)

[17] Bertin M C, Pimentel B M and Valcárcel C E 2008 Non-involutive constrained systems and Hamilton-Jacobi formalism Ann. Phys. 3233137

[18] Bertin M C, Pimentel B M and Valcárcel C E 2014 Involutive constrained systems and HamiltonJacobi formalism J. Math. Phys. 55112901

[19] Pimentel B M, Texeira R G and Tomazelli J L 1998 Hamilton-Jacobi approach to Berezinian singular systems Ann. Phys. 26775

Bertin M C, Pimentel B M and Pompeia P J 2005 First order actions: a new view Mod. Phys. Lett. A20 2873

Bertin M C, Pimentel B M and Pompeia P J 2008 Hamilton-Jacobi approach for first order actions and theories with higher derivatives Ann. Phys. 323527

[20] Bertin M C, Pimentel B M, Valcárcel C E and Zambrano G R 2014 Topologically massive yangmills field: a Hamilton-Jacobi approach J. Math. Phys. 55042902

[21] Pimentel B M, Pompeia P J and da Rocha-Neto J F 2005 The Hamilton-Jacobi approach to teleparallelism Il Nuovo Cimento B 120981

Bertin M C, Pimentel B M and Pompeia P J 2010 General Relativity in two dimensions: a Hamilton-Jacobi constraint analysis, Ann. Phys. 3252499 
Bertin M C, Pimentel B M and Valcárcel C E 2011 Hamilton-Jacobi formalism for Linearized Gravity Class. Quantum Grav. 28175015

[22] Bertin M C, Pimentel B M and Valcárcel C E 2012 Two-dimensional background field gravity: A Hamilton-Jacobi analysis J. Math. Phys. 53102901

[23] Henneaux M and Teitelboim C 1994 Quantization of Gauge Systems (Princeton University Press)

[24] Castellani L 1982 Symmetries in constrained hamiltonian systems Ann. of Phys. 143357 